\title{
GENERALIZED JOIN-HEMIMORPHISMS ON BOOLEAN ALGEBRAS
}

\author{
SERGIO CELANI
}

Received 14 November 2001

\begin{abstract}
We introduce the notions of generalized join-hemimorphism and generalized Boolean relation as an extension of the notions of join-hemimorphism and Boolean relation, respectively. We prove a duality between these two notions. We will also define a generalization of the notion of Boolean algebra with operators by considering a finite family of Boolean algebras endowed with a generalized join-hemimorphism. Finally, we define suitable notions of subalgebra, congruences, Boolean equivalence, and open filters.
\end{abstract}

2000 Mathematics Subject Classification: 06E25, 03 G99.

1. Introduction. In [4], Halmos generalizes the notion of Boolean homomorphism introducing the notion of join-hemimorphism between two Boolean algebras. A join-hemimorphism is a mapping between two Boolean algebras preserving 0 and $\vee$. As it is shown by Halmos in [4] and by Wright in [10], there exists a duality between join-hemimorphism and Boolean relations. On the other hand, Jónsson and Tarski in [5, 6] introduce the class of Boolean algebras with operators (BAO). They showed that a Boolean algebra endowed with a family of operators can be represented as a subalgebra of a power algebra $\mathscr{P}(X)$, where the operators of $\mathscr{P}(X)$ are defined by means of certain finitary relations on $X$. This class of algebras plays a key role in modal logic, and has very important applications in theoretical computer science (see, e.g., [1, 2]). The Halmos-Wright duality can be extended to a duality between BAO and Boolean spaces endowed with a set of finitary relations, which are a generalization of the Boolean relations. The aim of this paper is the study of an extension of these dualities.

In Section 2, we recall some notions on Boolean duality. In Section 3, we define the notion of generalized join-hemimorphism as a mapping between a finite product of a family of Boolean algebras $\left\{B_{1}, \ldots, B_{n}\right\}$ into a Boolean algebras $B_{0}$ such that it preserves 0 and $\vee$ in each coordinate. We will prove that there exists a duality between generalized join-hemimorphism and certain $(n+1)$-ary relations called generalized Boolean relations. This duality extends the duality given by Halmos and Wright. In Section 4 , we define the generalized modal algebra as a pair $\left\langle\left\{B_{0}, B_{1}, \ldots, B_{n}\right\}, \diamond\right\rangle$, where $B_{0}, B_{1}, \ldots, B_{n}$ are Boolean algebras and $\diamond: \prod_{i=1}^{n} B_{i} \rightarrow B_{0}$ is a generalized join-hemimorphism. 
In Section 5, we define the notions of generalized subalgebra and generalized Boolean equivalence and we prove that these notions are duals. Similarly, in Section 6, we introduce the generalized congruences and generalized open filters and we will prove that there exists a bijective correspondence between them.

2. Preliminaries. A topological space is a pair $\langle X, O(X)\rangle$, or $X$, for short, where $\mathscr{O}(X)$ is a subset of $\mathscr{P}(X)$ that is closed under finite intersections and arbitrary unions. The set $O(X)$ is called the set of open sets of the topological space. The collection of all closed sets of a topological space $\langle X, \mathscr{O}(X)\rangle$ is denoted by $\mathscr{C}(X)$. The set $\operatorname{Clop}(X)$ is the set of closed and open sets of $\langle X, \mathcal{O}(X)\rangle$.

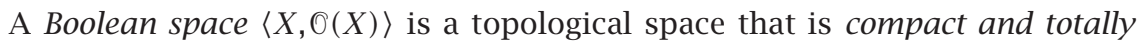
disconnected, that is, given distinct points $x, y \in X$, there is a clopen subset $U$ of $X$ such that $x \in U$ and $y \notin U$. If $\langle X, \mathcal{O}(X)\rangle$ is a Boolean space, then $\operatorname{Clop}(X)$ is a basis for $X$ and is a Boolean algebra under set-theoretical complement and intersection. Also, the application

$$
H_{X}: X \rightarrow U l(\operatorname{Clop}(X))
$$

given by $H_{X}(x)=\{U \in \operatorname{Clop}(X): x \in U\}$, is a bijective and continuous function. To each Boolean algebra $A$, we can associate a Boolean space $\operatorname{Spec}(A)$ whose points are the elements of $U l(A)$ with the topology determined by the clopen basis $\beta_{A}(A)=\left\{\beta_{A}(a): a \in A\right\}$, where $\beta_{A}: A \rightarrow \mathscr{P}(U l(A))$ is the Stone mapping defined by

$$
\beta_{A}(a)=\{P \in U l(A): a \in P\}
$$

By the above considerations, we have that, if $X$ is a Boolean space, then $X \cong$ $\operatorname{Spec}(\operatorname{Clop}(X))$, and if $A$ is a Boolean algebra, then $A \cong \operatorname{Clop}(\operatorname{Spec}(A))$.

Let $B$ be a Boolean algebra. The filter (ideal) generated by a set $H \subseteq A$ will be denoted by $F(H)(I(H))$. The lattice of all filters (ideals) of $B$ is denoted by $\operatorname{Fi}(A)(\operatorname{Id}(A))$.

Let $Y$ be a subset of a set $X$. The theoretical complement of $Y$ is denoted by $Y^{c}=X-Y$.

\section{Generalized join-hemimorphisms}

DEFINITION 3.1. Let $B_{0}, B_{1}, \ldots, B_{n}$ be Boolean algebras. A generalized joinhemimorphism is a function $\diamond: \prod_{i=1}^{n} B_{i} \rightarrow B_{0}$ such that

(1) $\diamond\left(a_{1}, \ldots, a_{i-1}, 0, a_{i+1}, \ldots, a_{n}\right)=0$,

(2) $\diamond\left(a_{1}, \ldots, a_{i-1}, x \vee y, a_{i+1}, \ldots, a_{n}\right)=\diamond\left(a_{1}, \ldots, a_{i-1}, x, a_{i+1}, \ldots, a_{n}\right) \vee$ $\diamond\left(a_{1}, \ldots, a_{i-1}, y, a_{i+1}, \ldots, a_{n}\right)$. 
It is easy to see that each generalized join-hemimorphism $h: \prod_{i=1}^{n} B_{i} \rightarrow B_{0}$ is monotonic in each variable, that is, $x, y \in B_{i}, x \leq y$, then

$$
\diamond\left(a_{1}, \ldots, x, \ldots, a_{n}\right) \leq \diamond\left(a_{1}, \ldots, y, \ldots, a_{n}\right) .
$$

A generalized join-hemimorphism $h: \prod_{i=1}^{n} B_{i} \rightarrow B_{0}$ defines a generalized meethemimorphism $\square: \prod_{i=1}^{n} B_{i} \rightarrow B_{0}$ as follows:

$$
\square\left(a_{1}, a_{2}, \ldots, a_{n}\right)=\neg \diamond\left(\neg a_{1}, \neg a_{2}, \ldots, \neg a_{n}\right) .
$$

It is clear that $\square$ preserves 1 and $\wedge$, and is monotonic in each variable.

Let $B_{0}, B_{1}, \ldots, B_{n}$ be Boolean algebras and let $\diamond: \prod_{i=1}^{n} B_{i} \rightarrow B_{0}$ be a generalized join-hemimorphism. Let $\vec{F}=F_{1} \times F_{2} \times \cdots \times F_{n}$, where $F_{i}$ is a filter of $B_{i}$, for $1 \leq i \leq n$. We consider the set

$$
\diamond(\vec{F})=\left\{y \in B_{0}:\left(\exists x_{i} \in F_{i}\right)\left(\diamond\left(x_{1}, x_{2}, \ldots, x_{n}\right) \leq y\right)\right\} .
$$

THEOREM 3.2. Let $B_{0}, B_{1}, \ldots, B_{n}$ be Boolean algebras and let $\diamond: \prod_{i=1}^{n} B_{i} \rightarrow B_{0}$ be a generalized join-hemimorphism. Let $F_{i}$ be a proper filter of $B_{i}$, for $1 \leq i \leq n$. Then

(1) $\diamond(\vec{F})$ is a proper filter of $B_{0}$,

(2) if $P \in U l\left(B_{0}\right)$ and $\diamond(\vec{F}) \subseteq P$, then there exist $Q_{i} \in U l\left(B_{i}\right)$, for $1 \leq i \leq n$ such that

$$
F_{i} \subseteq Q_{i}, \quad Q_{1} \times Q_{2} \times \cdots \times Q_{n} \subseteq \diamond^{-1}(P)
$$

PROoF. (1) It is easy to take into account that the function $\diamond$ is monotonic in each variable.

(2) Consider the family

$$
\mathscr{F}_{1}=\left\{Q_{1} \in F_{i}\left(B_{1}\right): F_{1} \subseteq Q_{1}, \diamond\left(Q_{1} \times F_{2} \times \cdots \times F_{n}\right) \subseteq P\right\} .
$$

We note that $\mathscr{F}_{1} \neq \varnothing$ because $F_{1} \in \mathscr{F}_{1}$. By Zorn's lemma, there exists a maximal element $Q_{1}$ in $\mathscr{F}_{1}$. We prove that $Q_{1} \in U l\left(B_{1}\right)$. Let $a \in B_{1}$ and suppose that $a$, $\neg a \notin Q_{1}$. Consider the filter $F_{a}=F\left(Q_{1} \cup\{a\}\right)$ and $F_{\neg a}=F\left(Q_{1} \cup\{\neg a\}\right)$. Since $q_{1}$ is maximal in $\mathscr{F}_{1}$, then $F_{a}, F_{\neg a} \notin \mathscr{F}_{1}$. So, there exist $\left(x_{1}, x_{2}, \ldots, x_{n}\right) \in F_{a} \times F_{2} \times$ $\cdots \times F_{n}$ and $\left(y_{1}, y_{2}, \ldots, y_{n}\right) \in F_{\neg a} \times F_{2} \times \cdots \times F_{n}$ such that $\diamond\left(x_{1}, x_{2}, \ldots, x_{n}\right) \notin P$ and $\diamond\left(y_{1}, y_{2}, \ldots, y_{n}\right) \notin P$. Since $x_{1} \in F_{a}$ and $y_{1} \in F_{\neg a}$, then $q_{1} \wedge a \leq x_{1}$ and $q_{2} \wedge \neg a \leq y_{1}$, for some $q_{1}, q_{2} \in Q_{1}$. As $Q_{1}$ is a filter of $B_{1}, q=q_{1} \wedge q_{2} \in Q_{1}$, $q \wedge a \leq x_{1}$, and $q \wedge \neg a \leq y_{1}$, and as each $F_{i}$ is a filter, we get $\mathrm{z}_{i}=x_{i} \wedge y_{i} \in F_{i}$, for $i=2, \ldots, n$. Then

$$
\begin{aligned}
\diamond\left(q, z_{2}, \ldots, z_{n}\right) & =\diamond\left(q \wedge(a \vee \neg a), z_{2}, \ldots, z_{n}\right) \\
& =\diamond\left((q \wedge a) \vee(q \wedge \neg a), z_{2}, \ldots, z_{n}\right) \\
& =\diamond\left(q \wedge a, z_{2}, \ldots, z_{n}\right) \vee \diamond\left(q \wedge \neg a, z_{2}, \ldots, z_{n}\right) \in P .
\end{aligned}
$$


If $\diamond\left(q \wedge a, z_{2}, \ldots, z_{n}\right) \in P$, then

$$
\diamond\left(q \wedge a, z_{2}, \ldots, z_{n}\right) \leq \diamond\left(q_{1} \wedge a, x_{2}, \ldots, x_{n}\right) \leq \diamond\left(x_{1}, x_{2}, \ldots, x_{n}\right) \in P,
$$

which is a contradiction. If $\diamond\left(q \wedge \neg a, z_{2}, \ldots, z_{n}\right) \in P$, then we deduce that $\diamond\left(y_{1}, y_{2}, \ldots, y_{n}\right) \in P$, which also is a contradiction. Thus, $a \in Q_{1}$ or $\neg a \in Q_{1}$.

Suppose that we have determinate ultrafilters $Q_{1}, \ldots, Q_{k}$ in $B_{1}, \ldots, B_{k}$, respectively, such that $F_{i} \subseteq Q_{i}$, for $1 \leq i \leq k$, and $\diamond\left(Q_{1} \times \cdots \times Q_{k} \times \cdots \times F_{n}\right) \subseteq P$. Consider the set

$$
\mathscr{F}_{k+1}=\left\{Q_{k+1} \in B_{k+1}: F_{i} \subseteq Q_{i}, \diamond\left(Q_{1} \times \cdots \times Q_{k+1} \times \cdots \times F_{n}\right) \subseteq P\right\} .
$$

We note that $\mathscr{F}_{k+1} \neq \varnothing$ because $F_{k+1} \in \mathscr{F}_{k+1}$. By the Zorn's lemma, there exists a maximal element $Q_{k+1}$ in $\mathscr{F}_{k+1}$. As in the above case, we can prove that $Q_{k+1} \in U l\left(B_{k+1}\right)$.

Therefore, we have ultrafilters $Q_{1}, \ldots, Q_{n}$ in $B_{1}, \ldots, B_{n}$, respectively, such that $F_{i} \subseteq Q_{i}$ and $\diamond\left(Q_{1} \times Q_{2} \times \cdots \times Q_{n}\right) \subseteq P$. It is easy to check that the last inclusion implies that $Q_{1} \times Q_{2} \times \cdots \times Q_{n} \subseteq \diamond^{-1}(P)$.

EXAMPLE 3.3. Let $X_{0}, \ldots, X_{n}$ be sets. Let $R \subseteq \prod_{i=0}^{n} X_{i}$. Then, the function $\diamond_{R}: \mathscr{P}\left(X_{1}\right) \times \cdots \times \mathscr{P}\left(X_{n}\right) \rightarrow \mathscr{P}\left(X_{0}\right)$, defined by

$$
\diamond_{R}\left(U_{1}, U_{2}, \ldots, U_{n}\right)=\left\{x_{0} \in X_{0}: R\left(x_{0}\right) \cap\left(U_{1} \times U_{2} \times \cdots \times U_{n}\right) \neq \varnothing\right\},
$$

where $R\left(x_{0}\right)=\left\{\left(x_{1}, \ldots, x_{n}\right) \in \prod_{i=1}^{n} X_{i}:\left(x_{0}, x_{1}, \ldots, x_{n}\right) \in R\right\}$, is a generalized join-homomorphism.

Definition 3.4. Let $X_{0}, \ldots, X_{n}$ be Boolean spaces. Consider a relation $R \subseteq$ $\prod_{i=0}^{n} X_{i}$. Then $R$ is a generalized Boolean relation if

(1) $R(x)$ is a closed subset in the product topology of $X_{1} \times \cdots \times X_{n}$, for each $x \in X_{0}$;

(2) for all $U_{i} \in \operatorname{Clop}\left(X_{i}\right)$, with $1 \leq i \leq n, \diamond_{R}\left(U_{1}, \ldots, U_{n}\right) \in \operatorname{Clop}\left(X_{0}\right)$.

We note that if $1 \leq i \leq 2$, then we have the notion of Boolean relation as defined in [4].

THEOREM 3.5. Let $B_{0}, B_{1}, \ldots, B_{n}$ be Boolean algebras and let $\diamond: \prod_{i=1}^{n} B_{i} \rightarrow$ $B_{0}$ be a generalized join-hemimorphism. Then the relation $R_{\diamond} \subseteq \prod_{i=0}^{n} U l\left(B_{i}\right)$, defined by

$$
\left(P, P_{1}, \ldots, P_{n}\right) \in R_{\diamond} \Longleftrightarrow P_{1} \times \cdots \times P_{n} \subseteq \diamond^{-1}(P),
$$


is a generalized Boolean relation such that

$$
\beta_{B_{0}}\left(\diamond\left(a_{1}, \ldots, a_{n}\right)\right)=\diamond_{R_{\diamond}}\left(\left(\beta_{B_{1}}\left(a_{1}\right), \ldots, \beta_{B_{n}}\left(a_{n}\right)\right)\right),
$$

for all $\left(a_{1}, \ldots, a_{n}\right) \in \prod_{i=1}^{n} B_{i}$.

Proof. We prove that for $P \in U l\left(B_{0}\right)$,

$$
R_{\diamond}(P)=\bigcap\left\{\left(\beta_{B_{1}}\left(a_{1}\right), \ldots, \beta_{B_{n}}\left(a_{n}\right)\right)^{c}: \diamond\left(a_{1}, \ldots, a_{n}\right) \notin P\right\} .
$$

If $\left(P_{1}, \ldots, P_{n}\right) \in R_{\diamond}(P)$ and

$$
\left(P_{1}, \ldots, P_{n}\right) \notin \bigcap\left\{\left(\beta_{B_{1}}\left(a_{1}\right), \ldots, \beta_{B_{n}}\left(a_{n}\right)\right)^{c}: \diamond\left(a_{1}, \ldots, a_{n}\right) \notin P\right\},
$$

then, for some $\diamond\left(a_{1}, \ldots, a_{n}\right) \notin P$, we get $\left(P_{1}, \ldots, P_{n}\right) \in\left(\beta_{B_{1}}\left(a_{1}\right), \ldots, \beta_{B_{n}}\left(a_{n}\right)\right)$, that is, $a_{i} \in P_{i}$ for $1 \leq i \leq n$. It follows that $\diamond\left(a_{1}, \ldots, a_{n}\right) \in P$, which is a contradiction. The other direction is similar. Thus, $R_{\diamond}(P)$ is a closed subset of $\prod_{i=1}^{n} U l\left(B_{i}\right)$.

Equality (3.11) follows by Theorem 3.2.

We note that the relation $R_{\diamond} \subseteq \prod_{i=0}^{n} U l\left(B_{i}\right)$ defined in Theorem 3.5 also can be defined using the notion of generalized meet-hemimorphism $\square$ in the following way:

$$
\left(P, P_{1}, \ldots, P_{n}\right) \in R_{\diamond} \Longleftrightarrow \square^{-1}(P) \subseteq P_{1}+\cdots+P_{n},
$$

where $P_{1}+\cdots+P_{n}=\left\{\left(a_{1}, \ldots, a_{n}\right) \in \prod_{i=1}^{n} B_{i}: a_{i} \in P_{i}\right.$, for some $\left.1 \leq i \leq n\right\}$.

LEMMA 3.6. Let $X_{0}, \ldots, X_{n}$ be Boolean spaces. Consider a relation $R \subseteq$ $\prod_{i=0}^{n} X_{i}$. Suppose that for all $U_{i} \in \operatorname{Clop}\left(X_{i}\right)$, with $1 \leq i \leq n, \diamond_{R}\left(U_{1}, \ldots, U_{n}\right) \in$ $\operatorname{Clop}\left(X_{0}\right)$. Then the following conditions are equivalent:

(1) $R$ is a generalized Boolean relation;

(2) if $\left(H_{x_{0}}\left(x_{0}\right), \ldots, H_{X_{n}}\left(x_{n}\right)\right) \in R_{\diamond_{R}}$, then $\left(x_{0}, \ldots, x_{n}\right) \in R$.

Proof. $(1) \Rightarrow(2)$. Suppose that $\left(H_{X_{0}}\left(x_{0}\right), \ldots, H_{X_{n}}\left(x_{n}\right)\right) \in R_{\diamond_{R}}$ and $\left(x_{0}, \ldots, x_{n}\right)$ $\notin R$. Since $R\left(x_{0}\right)$ is a closed subset of $\prod_{i=1}^{n} X_{i}$, there exist $U_{i} \in \operatorname{Clop}\left(X_{i}\right)$ such that $R\left(x_{0}\right) \cap\left(U_{1}, \ldots, U_{n}\right)=\varnothing$ and $x_{i} \in U_{i}$. Then, $x_{0} \notin \diamond_{R}\left(\left(U_{1}, \ldots, U_{n}\right)\right)$, that is, $\left(U_{1}, \ldots, U_{n}\right) \notin H_{X_{1}}\left(x_{1}\right) \times \cdots \times H_{X_{n}}\left(x_{n}\right)$, which is a contradiction.

$(2) \Rightarrow(1)$. We have to prove that $R(x)$ is a closed subset of $X_{0} \times \cdots \times X_{1}$. Suppose that $\left(x_{1}, \ldots, x_{n}\right) \notin R(x)$. Then, $\left(H_{X_{0}}(x), \ldots, H_{X_{n}}\left(x_{n}\right)\right) \notin R_{\diamond_{R}}$, that is, for each $1 \leq i \leq n$, there exist $U_{i} \in D_{i}$ such that $R(x) \cap\left(U_{1}, \ldots, U_{n}\right)=\varnothing$ and $x_{i} \in U_{i}$. Thus, $R(x)$ is a closed subset. 
By the above results, we deduce that there exists a duality between generalized Boolean relations and generalized join-hemimorphisms.

THeOREM 3.7. Let $X_{0}, \ldots, X_{n}$ be Boolean spaces. Let $R \subseteq \prod_{i=0}^{n} X_{i}$ be a generalized Boolean relation. Then the mapping $\diamond_{R}: \operatorname{Clop}\left(X_{1}\right) \times \cdots \times \operatorname{Clop}\left(X_{n}\right) \rightarrow$ Clop $\left(X_{0}\right)$, defined as in Example 3.3, is a generalized join-hemimorphism such that $\left(H_{x_{0}}\left(x_{0}\right), \ldots, H_{X_{n}}\left(x_{n}\right)\right) \in R_{\diamond_{R}}$ if and only if $\left(x_{0}, \ldots, x_{n}\right) \in R$, for all $\left(x_{0}, \ldots, x_{n}\right) \in \prod_{i=0}^{n} X_{i}$.

PRoof. It is clear that if $\left(x_{0}, \ldots, x_{n}\right) \in R$, then $\left(H_{x_{0}}\left(x_{0}\right), \ldots, H_{X_{n}}\left(x_{n}\right)\right) \in$ $R_{\diamond_{R}}$. The other direction follows by Lemma 3.6.

As an application of the above duality, we prove a generalization of the result that asserts that the Boolean homomorphisms are the minimal elements in the set of all join-hemimorphisms between two Boolean algebras (see [3]).

Let $\left\{B_{i}\right\}=\left\{B_{0}, \ldots, B_{n}\right\}$ be a family of Boolean algebras. Let $\mathrm{GJH}\left(\prod_{i=1}^{n} B_{i}, B_{0}\right)$ be the set of all generalized join-hemimorphisms between $\prod_{i=1}^{n} B_{i}$ and $B_{0}$ endowed with the pointwise order. Similarly, let $\operatorname{GBR}\left(\prod_{i=0}^{n} X_{i}\right)$ be the set of all generalized Boolean relations defined in $\prod_{i=0}^{n} X_{i}$ endowed with the pointwise order. Let $\diamond_{1}$ and $\diamond_{2} \in \mathrm{GJH}\left(\prod_{i=1}^{n} B_{i}, B_{0}\right)$ and let $R_{\diamond_{1}}$ and $R_{\diamond_{2}} \in \mathrm{GBR}\left(\prod_{i=0}^{n} U l\left(B_{i}\right)\right)$ be the associate generalized Boolean relations. It is clear that $\diamond_{1} \leq \diamond_{2}$ if and only if $R_{\diamond_{1}} \subseteq R_{\diamond_{2}}$.

THEOREM 3.8. An element of $\operatorname{GBR}\left(\prod_{i=0}^{n} X_{i}\right)$ is minimal if and only if it is a continuous function.

Proof. Let $R \subseteq \prod_{i=0}^{n} X_{i}$ be a minimal element in $\operatorname{GBR}\left(\prod_{i=0}^{n} X_{i}\right)$. We prove that it is a function. Let $x \in X_{0}$ and let $\vec{x}, \vec{y} \in \prod_{i=1}^{n} X_{i}$ such that $\vec{x}, \vec{y} \in R(x)$. Suppose that $\vec{x} \neq \vec{y}$. Then, $x_{i} \neq y_{i}$ for some $1 \leq i \leq n$. Then, there exist $U_{i} \in \operatorname{Clop}\left(X_{i}\right)$ such that $x_{i} \in U_{i}$ and $y_{i} \notin U_{i}$. Consider the sequence $\vec{U}=$ $\left(X_{1}, \ldots, U_{i}, \ldots, X_{n}\right)$. Then, $\vec{x} \in \vec{U}$ and $\vec{y} \notin \vec{U}$. We define an auxiliary relation $R_{\vec{U}} \subseteq \prod_{i=0}^{n} X_{i}$ by

$$
R_{\vec{U}}(x)= \begin{cases}R(x), & \text { if } x \notin \diamond_{R}(\vec{U}), \\ R(x) \cap \vec{U}, & \text { if } x \in \diamond_{R}(\vec{U}) .\end{cases}
$$

We prove that $R_{\vec{U}}$ is a generalized Boolean relation. It is clear that $R_{\vec{U}}$ is closed. Let $\vec{V}=\left(V_{1}, \ldots, V_{n}\right) \in \operatorname{Clop}\left(X_{1}\right) \times \cdots \times \operatorname{Clop}\left(X_{n}\right)$. Then

$$
\begin{aligned}
\diamond_{R_{\vec{U}}}(\vec{V})= & \left\{x \in X_{0}: R_{\vec{U}}(x) \cap \vec{V} \neq \varnothing\right\} \\
= & \left\{x \in X_{0}: x \in \diamond_{R}(\vec{U}), R(x) \cap \vec{U} \cap \vec{V} \neq \varnothing\right\} \\
& \cup\left\{x \in X_{0}: x \notin \diamond_{R}(\vec{U}), R(x) \cap \vec{V} \neq \varnothing\right\} \\
= & \diamond_{R}(\vec{U} \cap \vec{V}) \cup \diamond_{R}(\vec{V}) \cap \diamond_{R}(\vec{U})^{c} \in \operatorname{Clop}\left(X_{0}\right) .
\end{aligned}
$$


Thus, $R_{\vec{U}}$ is a generalized Boolean relation. It is clear that $R_{\vec{U}} \subset R$, that is, $R$ is not a minimal element in $\operatorname{GBR}\left(\prod_{i=0}^{n} X_{i}\right)$, which is a contradiction. Thus, $R$ is a continuous function.

If $R$ is continuous function, then it is easy to see that $R$ is a minimal element in $\operatorname{GBR}\left(\prod_{i=0}^{n} X_{i}\right)$.

4. Generalized modal algebras. Now we consider a finite family of Boolean algebras endowed with a generalized join-hemimorphism. This class of structures is a generalization of the notion of Boolean algebra with operators. In the sequel, we will write $\left\{X_{i}\right\}$ to denote the family of sets $\left\{X_{i}: 1 \leq i \leq n\right\}$.

Definition 4.1. Let $\left\{B_{i}\right\}$ be a family of Boolean algebras. A generalized modal algebra is a pair $B=\left\langle\left\{B_{i}\right\}, \diamond\right\rangle$, where $\diamond: \prod_{i=1}^{n} B_{i} \rightarrow B_{0}$ is a generalized join-hemimorphism.

Definition 4.2. A generalized modal space is a structure $\mathscr{F}=\left\langle\left\{X_{i}\right\}, R\right\rangle$, where $X_{0}, X_{1}, \ldots, X_{n}$ are Boolean spaces and $R$ is generalized Boolean relation.

DEFINITION 4.3. Let $B=\left\langle\left\{B_{i}\right\}, \diamond_{B}\right\rangle$ and $A=\left\langle\left\{A_{i}\right\}, \diamond_{A}\right\rangle$ be two generalized modal algebras. A generalized homomorphism between $B$ and $A$ is a finite sequence $h=\left(h_{0}, h_{1}, \ldots, h_{n}\right)$ such that

(1) $h_{i}: B_{i} \rightarrow A_{i}$ is a Boolean homomorphism for each $1 \leq i \leq n$,

(2) $h_{0}\left(\diamond_{B}\left(a_{1}, \ldots, a_{n}\right)\right)=\diamond_{A}\left(h_{1}\left(a_{1}\right), \ldots, h_{n}\left(a_{n}\right)\right)$.

We write $h: B \rightarrow A$ to denote that there exists a generalized homomorphism $h$ between the generalized modal algebras $B$ and $A$. We say that a generalized homomorphism $h$ between two generalized modal algebras $A$ and $B$ is injective if each Boolean homomorphism $h_{i}, 1 \leq i \leq n$, is injective, and $h$ is surjective if each $h_{i}$ surjective. Finally, $h$ is a generalized isomorphism if $h$ is bijective generalized homomorphism.

THEOREM 4.4. Let $B=\left\langle\left\{B_{i}\right\}, \diamond\right\rangle$ be a generalized modal algebra. Then the structure $\mathscr{F}(B)=\left\langle\left\{U l\left(B_{i}\right)\right\}, R_{\diamond}\right\rangle$ is a generalized modal space such that $B$ is isomorphic to the generalized modal algebra $A\left(\mathscr{F}_{F}(B)\right)=\left\langle\left\{\operatorname{Clop}\left(U l\left(B_{i}\right)\right)\right\}, R_{\diamond_{R}}\right\rangle$.

Proof. It is clear that $R_{\diamond}$ is a generalized join-hemimorphism. By Theorem 3.5 we have that $\beta=\left(\beta_{B_{0}}, \beta_{B_{1}}, \ldots, \beta_{B_{n}}\right)$ is a generalized homomorphism, and since each $\beta_{B_{i}}: B_{i} \rightarrow \operatorname{Clop}\left(U l\left(B_{i}\right)\right)$, for $1 \leq i \leq n$, is a Boolean isomorphism, then $\beta$ is an generalized isomorphism.

DeFinITION 4.5. Let $\mathscr{F}_{F}=\left\langle\left\{X_{i}\right\}, R\right\rangle$ and $\mathscr{G}_{g}=\left\langle\left\{Y_{i}\right\}, S\right\rangle$ be two generalized modal spaces. A generalized morphism between $\mathscr{F}_{F}$ and $\mathscr{G}$ is a sequence $f=$ $\left(f_{0}, f_{1}, \ldots, f_{n}\right)$ such that

(1) $f_{i}: X_{i} \rightarrow Y_{i}$ are continuous functions,

(2) if $\left(x_{0}, x_{1}, \ldots, x_{n}\right) \in R$, then $\left(f_{0}\left(x_{0}\right), f_{i}\left(x_{1}\right), \ldots, f_{n}\left(x_{n}\right)\right) \in S$, 
(3) if $\left(f_{0}\left(x_{0}\right), y_{1}, \ldots, y_{n}\right) \in S$, then there exist $x_{i} \in X_{i}$, with $1 \leq i \leq n$, such that $\left(x_{0}, x_{1}, \ldots, x_{n}\right) \in R$ and $f_{i}\left(x_{i}\right)=y_{i}$ with $1 \leq i \leq n$.

We write $f: \mathscr{F} \rightarrow \mathscr{G}$ to denote that there exists a generalized morphism $f$ between the generalized modal spaces $\mathscr{F}$ and $\mathscr{G}$.

Proposition 4.6. Let $\mathscr{F}_{F}=\left\langle\left\{X_{i}\right\}, R\right\rangle$ and $\mathscr{G}_{g}=\left\langle\left\{Y_{i}\right\}, S\right\rangle$ be two generalized modal spaces. If $f: \mathscr{F}_{F} \rightarrow \mathcal{G}$ is a generalized morphism, then the map $A(f)$ : $A(\mathscr{G}) \rightarrow A(\mathscr{F})$, defined by $A(f)=\left(f_{0}^{-1}, \ldots, f_{n}^{-1}\right)$ with $f_{i}^{-1}: \operatorname{Clop}\left(Y_{i}\right) \rightarrow \operatorname{Clop}\left(X_{i}\right)$, is a generalized homomorphism.

Proof. It is clear that each map $f_{i}^{-1}: \operatorname{Clop}\left(Y_{i}\right) \rightarrow \operatorname{Clop}\left(X_{i}\right)$ is a Boolean homomorphism. Let $U_{i} \in \operatorname{Clop}\left(X_{i}\right), 1 \leq i \leq n$, and $x_{0} \in X_{0}$ such that $x_{0} \in$ $f_{0}^{-1}\left(\diamond_{S}\left(U_{1}, \ldots, U_{n}\right)\right)$. Then $S\left(f_{0}\left(x_{0}\right)\right) \cap\left(U_{1}, \ldots, U_{n}\right) \neq \varnothing$. It follows that there exist $y_{i} \in Y_{i}$, with $i=1, \ldots, n$, such that $\left(f_{0}\left(x_{0}\right), y_{1}, \ldots, y_{n}\right) \in S$. Since $f$ is a generalized morphism, there exist $x_{i} \in X_{i}$, with $i=1, \ldots, n$, such that $\left(x_{0}, x_{1}, \ldots, x_{n}\right)$ $\in R$ and $f_{i}\left(x_{i}\right)=y_{i}$. So, $x_{i} \in f_{i}^{-1}\left(U_{i}\right)$, with $1 \leq i \leq n$ and this implies that

$$
R\left(x_{0}\right) \cap\left(f_{1}^{-1}\left(U_{1}\right), \ldots, f_{n}^{-1}\left(U_{n}\right)\right) \neq \varnothing,
$$

that is, $x_{0} \in \diamond_{R}\left(f_{1}^{-1}\left(U_{1}\right), \ldots, f_{n}^{-1}\left(U_{n}\right)\right)$.

The other direction is easy and left to the reader.

THEOREM 4.7. Let $B=\left\langle\left\{B_{i}\right\}, \diamond_{B}\right\rangle$ and $A=\left\langle\left\{A_{i}\right\}, \diamond_{A}\right\rangle$ be two generalized modal algebras and let $h=\left(h_{0}, \ldots, h_{n}\right)$ be a generalized homomorphism. Then the sequence $\mathscr{F}(f)=\left(h_{0}^{-1}, \ldots, h_{n}^{-1}\right)$ is a generalized morphism between the dual spaces $\mathscr{A}(\mathscr{F}(A))$ and $\mathscr{A}(\mathscr{F}(B))$.

Proof. It is clear that each $h_{i}^{-1}: U l\left(B_{i}\right) \rightarrow U l\left(A_{i}\right)$ is a continuous function. We prove conditions (2) and (3) of Definition 4.5 .

(2) Let $\left(P_{0}, \ldots, P_{n}\right) \in R_{\diamond_{B}}$ and let $\left(a_{1}, \ldots, a_{n}\right) \in h_{1}^{-1}\left(P_{1}\right) \times \cdots \times h_{n}^{-1}\left(P_{n}\right)$. Since $P \times \cdots \times P_{n} \subseteq \diamond_{B}^{-1}\left(P_{0}\right)$,

$$
\diamond_{A}\left(h_{1}\left(a_{1}\right), \ldots, h_{n}\left(a_{n}\right)\right)=h_{0}\left(\diamond_{B}\left(a_{1}, \ldots, a_{n}\right)\right) \in P_{0} .
$$

So, $\left(a_{1}, \ldots, a_{n}\right) \in \diamond_{A}\left(h_{0}^{-1}\left(P_{0}\right)\right)$.

(3) Let $\left(h_{1}^{-1}\left(P_{0}\right), Q_{1}, \ldots, Q_{n}\right) \in R_{\diamond_{B}}$. We prove that

$$
\diamond_{A}\left(h_{1}\left(Q_{1}\right) \times \cdots \times h_{n}\left(Q_{n}\right)\right) \subseteq P_{0} .
$$

Let $q_{i} \in Q_{i}$, with $1 \leq i \leq n$, such that $\left(h_{1}\left(q_{1}\right), \ldots, h_{n}\left(q_{n}\right)\right) \notin \diamond_{A}^{-1}\left(P_{0}\right)$. Then

$$
\diamond_{A}\left(h_{1}\left(q_{1}\right), \ldots, h_{n}\left(q_{n}\right)\right)=h_{0}\left(\diamond_{B}\left(q_{1}, \ldots, q_{n}\right)\right) \notin P_{0},
$$


that is, $\left(q_{1}, \ldots, q_{n}\right) \notin \diamond_{B}^{-1}\left(h_{0}^{-1}\left(P_{0}\right)\right)$, which is a contradiction. Thus,

$$
\diamond_{A}\left(h_{1}\left(Q_{1}\right) \times \cdots \times h_{n}\left(Q_{n}\right)\right) \subseteq P_{0} .
$$

We consider the filter $F_{i}=F\left(h_{i}\left(Q_{i}\right)\right)$, for $1 \leq i \leq n$. Then it is clear that

$$
F_{1} \times F_{2} \times \cdots \times F_{n} \subseteq \diamond_{B}^{-1}\left(P_{0}\right)
$$

By Theorem 3.2, there exist ultrafilters $P_{i} \in U l\left(A_{i}\right)$, for $1 \leq i \leq n$, such that $F_{i} \subseteq P_{i}$ and $P_{1} \times P_{2} \times \cdots \times P_{n} \subseteq \diamond_{A}^{-1}\left(P_{0}\right)$. Since $h_{i}\left(Q_{i}\right) \subseteq F_{i} \subseteq P_{i}$, we get $Q_{i}=$ $h_{i}^{-1}\left(P_{i}\right), 1 \leq i \leq n$.

We denote by $\mathscr{G} M A$ the class of generalized modal algebras with generalized homomorphisms and denote by $\mathscr{G}_{\mathcal{M}} \mathscr{E}$ the class of generalized modal spaces with generalized morphism. By the above results and by the Boolean duality, we can say that the classes $\mathscr{G M A}$ and $\mathscr{G} \mathcal{M} \mathscr{E}$ are dually equivalents.

5. Generalized subalgebras. It is known that there exists a duality between Boolean subalgebras of a Boolean algebra $A$ and equivalence relations defined on the dual space $U l(A)$ [7]. The duality is given as follows. Let $X$ be a Boolean space and let $E$ be an equivalence relation on $X$. A subset $U \subseteq X$ is said to be $E$-closed if for any $x, y \in X$, such that $(x, y) \in E$ and $x \in U$, then $y \in U$, that is,

$$
U_{E}=\{y \in X:(x, y) \in E, x \in U\} \subseteq U
$$

A Boolean equivalence is an equivalence $E$ defined on $X$ such that, for any $x, y \in X$, if $(x, y) \notin E$, there exists an $E$-closed $U \in \operatorname{Clop}(X)$ such that $x \in$ $U$ and $y \notin U$. The Boolean subalgebra of $\operatorname{Clop}(X)$ associated with a Boolean equivalence $E$ is defined by

$$
B(E)=\left\{U \in \operatorname{Clop}(X): U_{E}=U\right\}
$$

If $A$ is a Boolean algebra and $B$ is a Boolean subalgebra of $A$, then the relation $E(B) \subseteq U l(A)^{2}$, given by

$$
(P, Q) \in E(B) \Longleftrightarrow P \cap B=Q \cap B,
$$

is a Boolean equivalence.

THEOREM 5.1 [7]. Let A be a Boolean algebra. Then there exists a dual orderisomorphism between Boolean subalgebras of $A$ and Boolean equivalences defined on $U l(A)$.

DEFINITION 5.2. Let $B=\left\langle\left\{B_{i}\right\}, \diamond\right\rangle$ be a generalized modal algebra. A subalgebra of $B$ is a sequence $A=\left(A_{0}, \ldots, A_{n}\right)$ such that, for each $0 \leq i \leq n, A_{i}$ is a subalgebra of $B_{i}$, and for each $\vec{a} \in \prod_{i=1}^{n} B_{i}$, we get $\diamond(\vec{a}) \in B_{0}$. 
DEFINITION 5.3. Let $\mathscr{F}=\left\langle\left\{X_{i}\right\}, R\right\rangle$ be a generalized modal spaces. A generalized Boolean equivalence is a sequence $E=\left(E_{0}, E_{1}, \ldots, E_{n}\right)$ such that, for each $0 \leq i \leq n, E_{i}$ is a Boolean equivalence of $X_{i}$, and if $(a, b) \in E_{0}$ and $\left(a, x_{1}, \ldots, x_{n}\right) \in R$, then there exists $\left(y_{1}, \ldots, y_{n}\right) \in \prod_{i=1}^{n} X_{i}$, such that $\left(b, y_{1}, \ldots\right.$, $\left.y_{n}\right) \in R$ and $\left(x_{i}, y_{i}\right) \in E_{i}$, for each $1 \leq i \leq n$.

THEOREM 5.4. Let $B=\left\langle\left\{B_{i}\right\}, \diamond\right\rangle$ be a generalized modal algebra. Then the following conditions are equivalent:

(1) $A=\left\langle\left\{A_{i}\right\}, \diamond\right\rangle$ is a subalgebra of $B$;

(2) the sequence $E_{A}=\left(E_{B_{0}}, E_{B_{1}}, \ldots, E_{B_{n}}\right)$ is a generalized Boolean equivalence.

Proof. $(1) \Rightarrow(2)$. Let $A=\left\langle\left\{A_{i}\right\}, \diamond\right\rangle$ be a subalgebra of $B$. Let $P_{0}, Q_{0} \in U l\left(B_{0}\right)$ such that $P_{0} \cap A_{0}=Q_{0} \cap A_{0}$ and let $\left(P_{0}, P_{1}, \ldots, P_{n}\right) \in R_{B}$. We consider the set $\diamond\left(F\left(P_{1} \cap A_{1}\right), \ldots, F\left(P_{n} \cap A_{n}\right)\right)$, where $F\left(P_{i} \cap A_{i}\right)$ is the filter generated by the set $P_{i} \cap A_{i}$. We prove that

$$
\diamond\left(F\left(P_{1} \cap A_{1}\right), \ldots, F\left(P_{n} \cap A_{n}\right)\right) \subseteq Q_{0}
$$

Let $\vec{a}=\left(a_{1}, \ldots, a_{n}\right)$ with $a_{i} \in P_{i} \cap A_{i}$, for $1 \leq i \leq n$. Since $\vec{a} \in P_{1} \times \cdots \times P_{n} \subseteq$ $\diamond^{-1}\left(P_{0}\right), \diamond \vec{a} \in P_{0}$. As $\vec{a} \in \prod_{i=1}^{n} A_{i}$ and $A$ is a subalgebra of $B$, then $\diamond \vec{a} \in P_{0} \in$ $A_{0}=Q_{0} \cap A_{0}$. Thus, $\diamond \vec{a} \in Q_{0}$ and (5.4) is valid. By Theorem 3.2, there exist ultrafilters $Q_{i} \in U l\left(B_{i}\right)$, for $1 \leq i \leq n$, such that $P_{i} \cap A_{i}=Q_{i} \cap A_{i}$. Therefore, $\left(Q_{0}, Q_{1}, \ldots, Q_{n}\right) \in R_{B}$ and $P_{i} \cap A_{i}=Q_{i} \cap A_{i}$ for each $1 \leq i \leq n$.

$(2) \Rightarrow(1)$. Suppose that $E_{A}=\left(E_{B_{0}}, E_{B_{1}}, \ldots, E_{B_{n}}\right)$ is a generalized Boolean equivalence. Let $\vec{a} \in \prod_{i=1}^{n} A_{i}$ and suppose that $\diamond \vec{a} \notin A_{0}$. Consider the set in $B_{0}$,

$$
\left(F(\diamond \vec{a}) \cap A_{0}\right) \cup\{\neg \diamond \vec{a}\}
$$

This set has the finite intersection property. Suppose the contrary. Then, there exists $x \in F(\diamond \vec{a}) \cap A_{0}$ such that $x \wedge \neg \diamond \vec{a}=0$. It follows that $\diamond \vec{a} \leq x \leq \diamond \vec{a}$, that is, $x=\diamond \vec{a} \in A_{0}$, which is a contradiction. Thus, the set (5.5) has the finite intersection property. So, there exists an ultrafilter $P_{0} \in U l\left(B_{0}\right)$ such that

$$
F(\diamond \vec{a}) \cap A_{0} \subseteq P_{0}, \quad \neg \diamond \vec{a} \in P_{0} .
$$

Consider the set

$$
\{\diamond \vec{a}\} \cup P_{0} \cap A_{0}
$$

This set has the finite intersection property, because in contrary case there exists $p \in P_{0} \cap A_{0}$ such that $\diamond \vec{a} \leq \neg p$. This implies that $\neg p \in F(\diamond \vec{a}) \cap A_{0} \subseteq P_{0}$, which is a contradiction. Thus, there exists $Q_{0} \in U l\left(B_{0}\right)$ such that

$$
\diamond \vec{a} \in Q_{0}, \quad P_{0} \cap A_{0}=Q_{0} \cap A_{0} .
$$


Since $\diamond \vec{a} \in Q_{0}$, there exists $\left(Q_{1}, \ldots, Q_{n}\right) \in \prod_{i=1}^{n} U l\left(B_{i}\right)$ such that

$$
\left(Q_{0}, Q_{1}, \ldots, Q_{n}\right) \in R_{B}, \quad \vec{a} \in\left(Q_{1}, \ldots, Q_{n}\right)
$$

By hypothesis, there exists $\left(P_{1}, \ldots, P_{n}\right) \in R_{B}\left(P_{0}\right)$ such that $P_{i} \cap A_{0}=Q_{i} \cap A_{0}$, for $1 \leq i \leq n$. Hence, $a_{i} \in Q_{i} \cap A_{0}$, we have $\diamond \vec{a} \in P_{0}$, which is a contradiction by (5.6). Thus, $\diamond \vec{a} \in A_{0}$.

6. Generalized congruences. Recall that, given a modal algebra $B$, there exists a bijective correspondence between congruences of $B$ and filters $F$ of $B$ closed under $\square$, that is, $\square a \in F$ when $a \in F$ (see, e.g., [8, 9]). This class of filters are called open filters. In this section, we introduce a generalization of the notion of congruences and open filter.

Let $B$ be a Boolean algebra. Recall that if $F$ is a filter of $B$, the relation

$$
\theta(F)=\{(x, y): \exists f: x \wedge f=y \wedge f\}
$$

is a Boolean congruence. On the other hand, if $\theta$ is a Boolean congruence, then

$$
F(\theta)=\{x \in B:(x, 1) \in \theta\}
$$

is a filter of $B$ such that $\theta(F(\theta))=\theta$ and $F(\theta(F))=F$.

Let $B=\left\langle\left\{B_{i}\right\}, \diamond\right\rangle$ be a generalized modal algebra, let $F_{i}$ be a filter of $B_{i}, 1 \leq i \leq$ $n$, and let $F_{1}+\cdots+F_{n}=\left\{\left(a_{1}, \ldots, a_{n}\right) \in \prod_{i=1}^{n} B_{i}: a_{i} \in F_{i}\right.$, for some $\left.1 \leq i \leq n\right\}$.

DEFINITION 6.1. Let $B=\left\langle\left\{B_{i}\right\}, \diamond\right\rangle$ be a generalized modal algebra. A generalized modal filter of $B$ is a sequence $\vec{F}=\left(F_{0}, F_{1}, \ldots, F_{n}\right)$ such that

(1) $F_{i}$ is a filter of $B_{i}, 0 \leq i \leq n$;

(2) for any $\vec{a} \in F_{1}+\cdots+F_{n}, \square \vec{a} \in F_{0}$.

DEFINITION 6.2. Let $B=\left\langle\left\{B_{i}\right\}, \diamond\right\rangle$ be a generalized modal algebra. A generalized modal congruence of $B$ is a finite sequence

$$
\theta=\left(\theta_{0}, \ldots, \theta_{n}\right)
$$

such that

(1) $\theta_{i}$ is a Boolean congruence of $B_{i}$, for each $0 \leq i \leq n$;

(2) if $\left(a_{i}, b_{i}\right) \in \theta_{i}$ with $1 \leq i \leq n$, then $\left(\diamond\left(a_{1}, \ldots, a_{n}\right), \diamond\left(b_{1}, \ldots, b_{n}\right)\right) \in \theta_{0}$.

THEOREM 6.3. Let $B=\left\langle\left\{B_{i}\right\}, \diamond\right\rangle$ be a generalized modal algebra. There exists a bijective correspondence between congruences of $B$ and the generalized modal filter of $B$. 
Proof. Let $\theta=\left(\theta_{0}, \ldots, \theta_{n}\right)$ be a generalized congruence of $B$. Define the sequence $F(\theta)=\left(F\left(\theta_{0}\right), \ldots, F\left(\theta_{n}\right)\right)$. Let $\left(a_{1}, a_{2}, \ldots, a_{n}\right) \in F\left(\theta_{1}\right)+\cdots+F\left(\theta_{n}\right)$. Then there exist some $1 \leq j \leq n$ such that $\left(a_{j}, 1\right) \in \theta_{j}$. Since $\left(a_{i}, a_{i}\right) \in \theta_{i}$ for every $1 \leq i \leq n$, then

$$
\left(\square\left(a_{1}, \ldots, a_{j}, \ldots, a_{n}\right), \square\left(a_{1}, \ldots, 1, \ldots, a_{n}\right)\right)=\left(\square\left(a_{1}, \ldots, a_{j}, \ldots, a_{n}\right), 1\right) \in \theta_{0} .
$$

Thus, $\square\left(a_{1}, \ldots, a_{j}, \ldots, a_{n}\right) \in F\left(\theta_{0}\right)$.

Let $\vec{F}=\left(F_{0}, F_{1}, \ldots, F_{n}\right)$ be a generalized modal filter. Consider the sequence $\theta(\vec{F})=\left(\theta\left(F_{0}\right), \ldots, \theta\left(F_{n}\right)\right)$. Let $\left(a_{i}, b_{i}\right) \in \theta\left(F_{i}\right)$, for $1 \leq i \leq n$. Then, for each $1 \leq i \leq n$, there exist $f_{i} \in F_{i}$ such that $a_{i} \wedge f_{i}=b_{i} \wedge f_{i}$. We prove that there exists $f_{0} \in F_{0}$ such that $\square\left(a_{1}, \ldots, a_{n}\right) \wedge f_{0}=\square\left(b_{1}, \ldots, b_{n}\right) \wedge f_{0}$. Suppose the contrary, that is, for every $f_{0} \in F_{0}$,

$$
\square\left(a_{1}, \ldots, a_{n}\right) \wedge f_{0} \neq \square\left(b_{1}, \ldots, b_{n}\right) \wedge f_{0} .
$$

Then there exists $P_{0} \in U l\left(A_{0}\right)$ such that

$$
F_{0} \subseteq P_{0}, \quad \square\left(a_{1}, \ldots, a_{n}\right) \in P_{0}, \quad \square\left(b_{1}, \ldots, b_{n}\right) \notin P_{0} .
$$

So, there exists $\left(P_{1}, \ldots, P_{n}\right) \in R_{B}\left(P_{0}\right)$ such that

$$
a_{i} \notin P_{i}, \quad \forall 1 \leq i \leq n .
$$

Since $\square\left(a_{1}, \ldots, a_{n}\right) \in P_{0}$, then $a_{j} \in P_{j}$, for some $1 \leq j \leq n$, and as $\left(0, \ldots, f_{j}, \ldots, 0\right)$ $\in F_{1}+\cdots+F_{n}$, we get $\square\left(0, \ldots, f_{j}, \ldots, 0\right) \in F_{0} \subseteq P_{0}$. It follows that $f_{j} \in P_{j}$ and since $a_{j} \wedge f_{j}=b_{j} \wedge f_{j}, b_{j} \in P_{j}$, which is a contradiction by (6.7). Thus, there exists $f_{0} \in F_{0}$ such that $\square\left(a_{1}, \ldots, a_{n}\right) \wedge f_{0}=\square\left(b_{1}, \ldots, b_{n}\right) \wedge f_{0}$.

\section{REFERENCES}

[1] M. M. Bonsangue and M. Z. Kwiatkowska, Re-interpreting the modal $\mu$-calculus, Modal Logic and Process Algebra. A Bisimulation Perspective (Amsterdam, 1994) (A. Ponse, M. de Rijke, and Y. Venema, eds.), CSLI Lecture Notes, vol. 53, CSLI Publications, California, 1995, pp. 65-83.

[2] C. Brink and I. M. Rewitzky, Finite-cofinite program relations, Log. J. IGPL 7 (1999), no. 2, 153-172.

[3] S. Graf, A selection theorem for Boolean correspondences, J. reine angew. Math. 295 (1977), 169-186.

[4] P. R. Halmos, Algebraic Logic, Chelsea Publishing, New York, 1962.

[5] B. Jónsson and A. Tarski, Boolean algebras with operators. I, Amer. J. Math. 73 (1951), 891-939.

[6] _ Boolean algebras with operators. II, Amer. J. Math. 74 (1952), 127-162.

[7] S. Koppelberg, Topological duality, Handbook of Boolean Algebras. Vol. 1 (J. D. Monk and R. Bonnet, eds.), North-Holland Publishing, Amsterdam, 1989, pp. 95-126.

[8] M. Kracht, Tools and Techniques in Modal Logic, Studies in Logic and the Foundations of Mathematics, vol. 142, North-Holland Publishing, Amsterdam, 1999. 
[9] G. Sambin and V. Vaccaro, Topology and duality in modal logic, Ann. Pure Appl. Logic 37 (1988), no. 3, 249-296.

[10] F. B. Wright, Some remarks on Boolean duality, Portugal. Math. 16 (1957), 109117.

Sergio Celani: Departamento de Matemática, Facultad de Ciencias Exactas, Universidad Nacional del Centro, 7000-Tandil, Provincia of Buenos Aires, Argentina

E-mail address: sce1ani@exa. unicen. edu . ar 


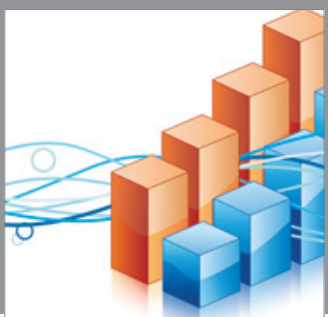

Advances in

Operations Research

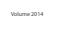

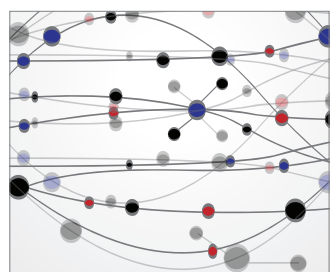

\section{The Scientific} World Journal
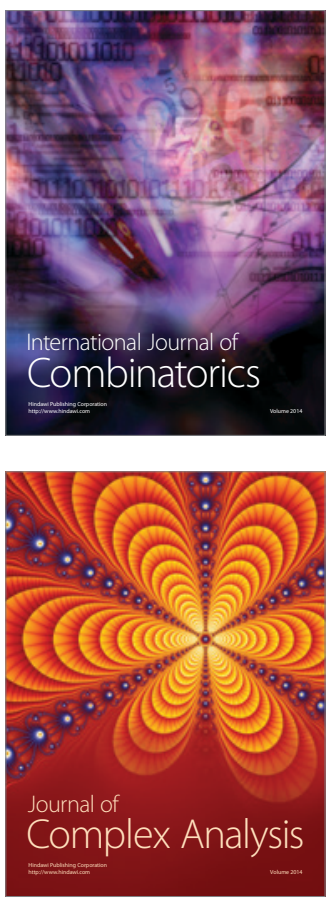

International Journal of

Mathematics and

Mathematical

Sciences
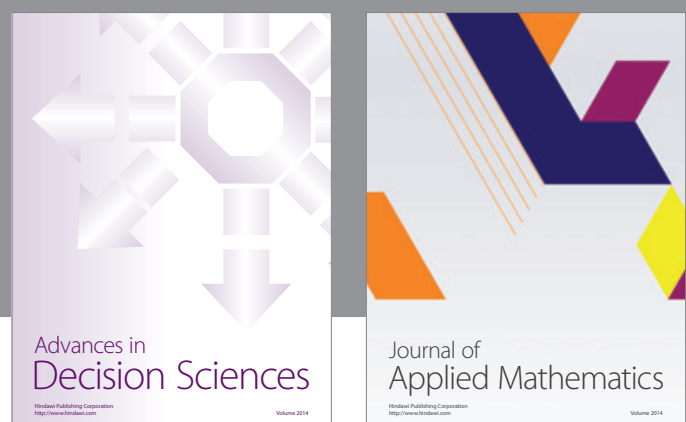

Journal of

Applied Mathematics
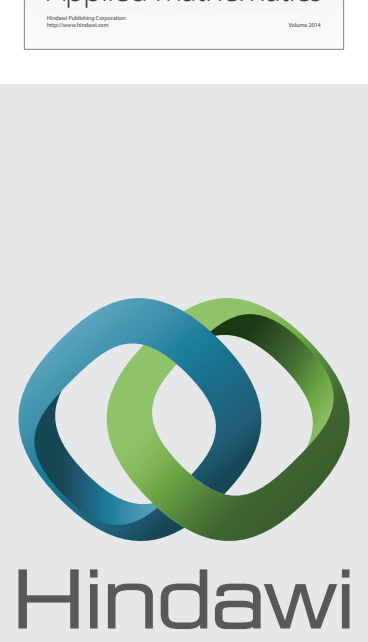

Submit your manuscripts at http://www.hindawi.com
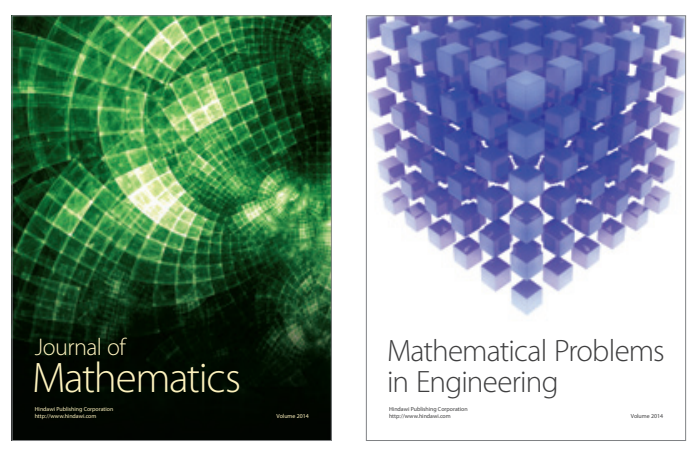

Mathematical Problems in Engineering
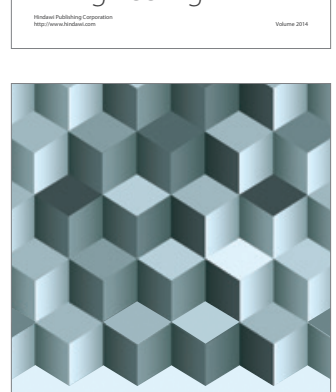

Journal of

Function Spaces
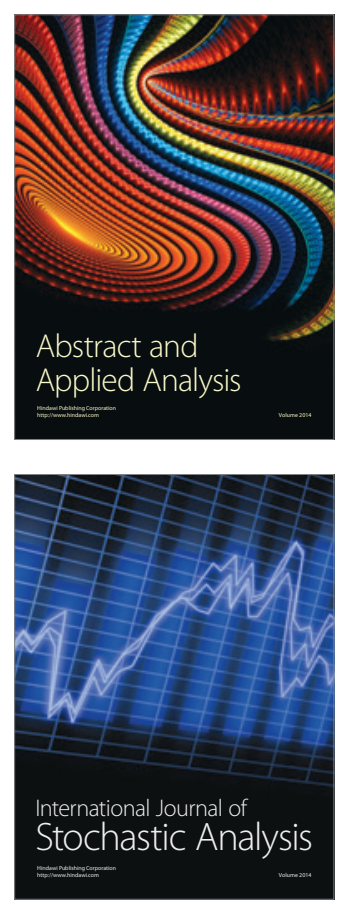

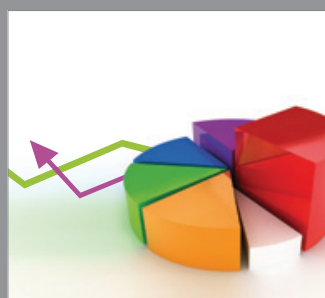

ournal of

Probability and Statistics

Promensencen
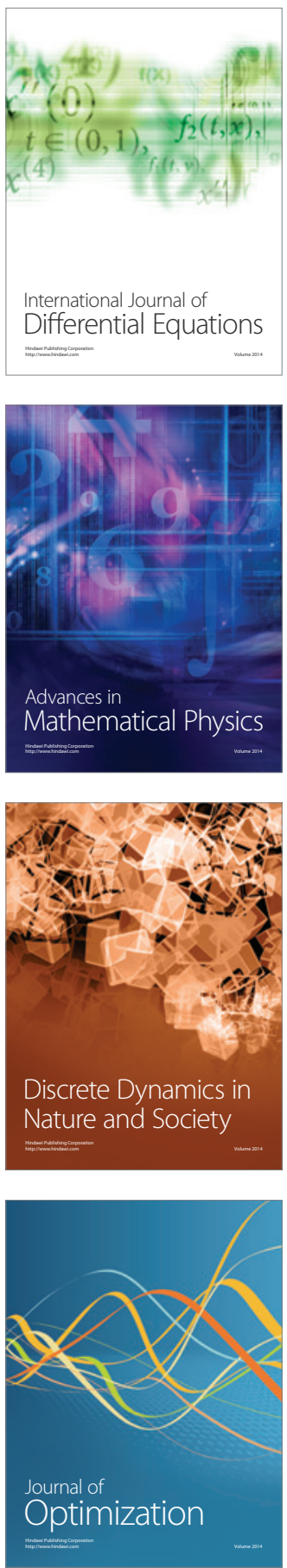